\title{
Prevalencia de gingivitis y factores asociados en niños chilenos de cuatro años
}

\section{Prevalence of gingivitis and associated factors in 4 year-old chilean children}

\section{Zaror Sánchez C*,**, Muñoz Millán P*, Sanhueza Campos A***}

\section{RESUMEN}

Objetivos: Determinar la prevalencia y distribución de gingivitis y su relación con factores socioeconómicos, biológicos y locales en niños chilenos de 4 años.

Metodología: Se realizó un estudio transversal en preescolares de 4 años que acuden al Hospital de Calbuco, Región de Los Lagos, Chile. La muestra consistió en 102 niños/niñas de 4 años. Un examinador, previamente calibrado, realizó examen odontológico, determinando presencia de placa bacteriana visible, caries dental y sangramiento gingival.

Resultados: Los resultados demostraron una prevalencia del 93,1\% gingivitis, siendo los sextantes IV y VI los más afectados, con un 90 y $92 \%$ de prevalencia respectivamente. Al realizar el análisis de la correlación de gingivitis con otros factores, resultó una correlación positiva con caries y con el nivel de placa bacteriana, por sextante. No hubo correlaciones significativas con género, maloclusión, frecuencia de cepillado y responsable del cepillado.

Conclusión: La alta prevalencia encontrada, los sextantes más afectadas y la escasa relación con datos de la anamnesis del niño/niña, deben ser consideradas en la educación que se le entrega al adulto responsable del cepillado, particularmente en la población de nivel socioeconómico bajo, quienes son más susceptible a las enfermedades bucales.

Palabras clave: Gingivitis, placa bacteriana, factores de riesgo, prevalencia.

\section{SUMMARY}

Objective: To determine the prevalence and distribution of gingivitis and its relation with socioeconomic, biological and local factors in Chilean children of 4 years-old.

Methodology: A cross-sectional study was performed in pre-school children of 4 years old attended in Calbuco Hospital, Los Lagos Region, Chile. The sample consisted of 102 children of 4 years-old. An examiner, previously calibrated, realized clinical examination determining presence of visible plaque, dental decay and gingival bleeding.

Results: The prevalence of gingivitis was $93.1 \%$, being the sextants IV and IV the most affected, with a $90 \%$ and $92 \%$ of prevalence respectively. To performance the analysis of the correlation of gingivitis with other factors, a positive correlation was found with caries and plaque level, by sextant. There weren't significant correlations with genre, malocclusion, frequency of brushing and responsible of the brushing.

Conclusion: The high prevalence founded, the sextants more affected and the little relation with data of the anamnesis of children, must be considered in the education that is given to the caregivers, particularly in the population of low socioeconomic level, who are more susceptible to oral diseases.

* Departamento de Odontología Integral, Facultad de Medicina. Universidad de La Frontera. Temuco. Chile.

** Servicio de Salud del Reloncaví, X Región. Chile.

*** Departamento de Matemáticas y Estadística, Universidad de la Frontera. Temuco. Chile. 
Key words: Gingivitis, plaque, factors of risk, prevalence.

Fecha de recepción: 15 de junio de 2010.

Aceptado para publicación: 27 de julio de 2010.

Zaror Sánchez C, Muñoz Millán P, Sanhueza Campos A. Prevalencia de gingivitis y factores asociados en niños chilenos de cuatro años. Av. Odontoestomatol 2012; 28 (1): 33-38.

\section{INTRODUCCIÓN}

La enfermedad periodontal es una de las patologías más prevalentes de la cavidad oral en todos los grupos etarios (1). Dentro de éstas, la gingivitis inducida por placa bacteriana es la más prevalente en niños y adolescentes (2-4). Si bien este tipo de gingivitis se caracteriza por ser causada por una infección bacteriana no específica, en niños se ha encontrado un aumento en los niveles de Actinomyces sp, Capnocytophaga sp, Leptotrichia sp. Selenomonas sp. al compararlo con la gingivitis en adultos (4-5).

La severidad de gingivitis es menos intensa en niños que en adultos (4). Ésta se inicia en la infancia seguida de un aumento gradual en su prevalencia alcanzando su peak en la pubertad y ocurriendo como es de esperarse primero en las mujeres (2, 6-10). Este incremento con la edad, es atribuido al aumento de los sitios de riesgo, la acumulación de placa asociada con la erupción y exfoliación dental y a la influencia de los factores hormonales durante la pubertad.

Durante la adolescencia existe una disminución en la prevalencia de la patología asociada a un aumento en la conciencia social y mejor higiene oral (2).

Si bien la presencia de placa bacteriana es el principal factor etiológico de la enfermedad en la dentición primaria, no existe relación entre la cantidad de placa y la intensidad de inflamación de los tejidos gingivales $(6,11-13)$.

Otros factores que pueden afectar la ocurrencia y severidad de gingivitis en niños son respuesta inmunológica de las células inflamatorias, cambios en la composición bacteriana de la placa dental, diferencias morfológicas en la dentición primaria, presencia de apiñamiento y traumatismo por cepillado factores demográficos, socioeconómicos y estrés (10, 13-16).

En relación a la epidemiología de la gingivitis esta varía de una población a otra con prevalecías que van desde un $25 \%$ a un $100 \%$. En Chile existen escasas investigaciones en niños, sólo existen reportes ministeriales no publicados con prevalencia de $6,2 \%$ a los 4 años (2, 7, 9, 17-19).

Los sitios gingivales más afectados por gingivitis son las superficies linguales de los molares inferiores seguido de las superficies bucales y palatinas de los maxilares superiores $(2,9,20)$.

El propósito de este estudio es evaluar la prevalencia y distribución de gingivitis en niños de 4 años con el fin de contribuir con información diagnóstica regional que nos permita conocer cómo se comporta la gingivitis en ésta población infantil y así tomar medidas programáticas y preventivas acorde a la realidad local.

\section{MATERIAL Y MÉTODO}

\section{Población}

Se realizó un estudio descriptivo, de corte transversal en el Hospital de Calbuco, perteneciente al Servicio Salud de Reloncaví, X región, Chile. Calbuco está ubicado a 56 kilómetros al sur oeste de Puerto Montt, capital de la décima región, es un archipiélago conformado por catorce islas que están unidas al continente por un pedrapién y su población mayoritariamente tiene un nivel socioeconómico medio-bajo. La población total de la comuna de Calbuco en niños de 4 años es de 568, (296 de sexo masculino y 
272 de sexo femenino), distribuyéndose en 234 del área urbana $(118=$ sexo masculino; $116=$ sexo femenino), y 334 del área rural (178 = sexo masculino; 156 = sexo femenino). De esta población de referencia el $72 \%$ (409 niños) son beneficiarios de los establecimientos públicos de salud.

La selección de la muestra fue determinada por método no probabilístico, de casos consecutivos. Se estudió un total de 102 niños de 4 años que corresponde a un $25 \%$ del total de niños beneficiarios de 4 años del Hospital de Calbuco, utilizando una prevalencia de gingivitis del 50\%, un error de muestreo del $9 \%$, un nivel de confianza del $95 \%$ y un tamaño de la población de 568 individuos, se obtiene un tamaño de muestra mínimo de 100 pacientes. Este cálculo se realizó haciendo uso del programa estadístico computacional nQuery Adviser.

Se excluyeron los niños con discapacidad, enfermedad sistémica de base, uso crónico de medicamentos y/o atención odontológica previa.

\section{Examen clínico}

Los niños fueron examinados por un solo investigador, calibrado previamente por prueba piloto y con un coeficiente de correlación intraclásica de un $98 \%$, en las dependencias del Servicio Dental, bajo luz artificial e inspección visual de la cavidad oral. Se utilizó una Ficha Clínica Odontológica diseñada para el estudio, que incluye antecedentes registrados por el examinador y otorgados por la madre.

La condición gingival fue evaluada mediante el índice de hemorragia gingival de Ainamo y Bay (GBI), el cual evalúa la hemorragia al sondaje. Fueron evaluadas todas las piezas dentarias presentes, para lo cual se utilizó una sonda Williams para obtener el GBI, la cual fue introducida suavemente alrededor de 0,5 $\mathrm{mm}$ en el surco gingival y movida a través de toda su extensión.

Se consideró presencia de gingivitis cuando un sujeto presentó a lo menos una superficie con hemorragia. El índice promedio para cada sujeto fue calculado como el porcentaje de superficies que presentaron hemorragia.
La presencia de caries se estableció en base a los criterios propuestos por la OMS en el Oral Health Survey Basic Methods, para estudios epidemiológicos.

El tipo de maloclusión se determinó clínicamente evaluando la presencia o ausencia de apiñamiento en el segmento anterior del maxilar superior e inferior y/o cualquier otro tipo de alteración.

\section{Descripción de las variables}

La ficha clínica registró datos demográficos, socioeconómicos y hábitos de higiene oral.

En relación a los hábitos higiénicos se registro la presencia de higiene oral, frecuencia de cepillado, responsable del cepillado y el índice de Higiene Oral Simplificado de Greene y Vermillion (IHOS) general y por sextante.

\section{Plan de análisis}

El análisis de los datos fue realizado por medio del programa estadístico stata 7.0. Se realizó un Modelo de Regresión para Medidas Repetidas de Factores Asociados a Gingivitis por sextante.

\section{RESULTADOS}

De los 102 niños de la muestra el 53,9\% de la muestra correspondió a hombres

Del total de 99 niños que se cepillan, el 3\% no se cepilla, el 10,1\% se cepilla 1 vez al día, en el 76,8\% la higiene es realizada 2 o 3 veces al día, y el 13,1\% lo realiza más de 3 veces al día. Por otro lado, del total de los 99 niños el 60,6\% lo hace por sí mismo, el $17,2 \%$ lo realiza un adulto y el $22,2 \%$ lo realiza el niño con ayuda de un adulto.

Del total de los niños que tienen hábitos de higiene, el $51,5 \%$ no se cepilla lo dientes antes de dormir.

En relación a la prevalencia de maloclusiones, el 2,9\% presenta apiñamiento y el $24,5 \%$ otro tipo de maloclusión. 
En el gráfico 1 se muestra la distribución de placa promedio por sextante, donde el sextante I y III presentan el mayor promedio de IHO-S que corresponde a 2,5.

La prevalencia de gingivitis encontrada en el grupo de estudio fue de un 93,1\%. El gráfico 2 muestra la prevalencia de gingivitis según sextantes, siendo el sextante IV él con mayor prevalencia con un $92 \%$.

La mayor prevalencia de gingivitis se produce en los sextantes posteroinferiores, seguidos por los posterosuperiores. La menor prevalencia de gingivitis se produce en el sextante anterosuperior.

Al realizar el modelo de regresión para medidas repetidas de los factores de riesgo asociados con gingivitis por sextante podemos observar que hay evidencia estadísticamente significativa para concluir que las variables caries y nivel de placa están asociadas con gingivitis (Tabla 1).

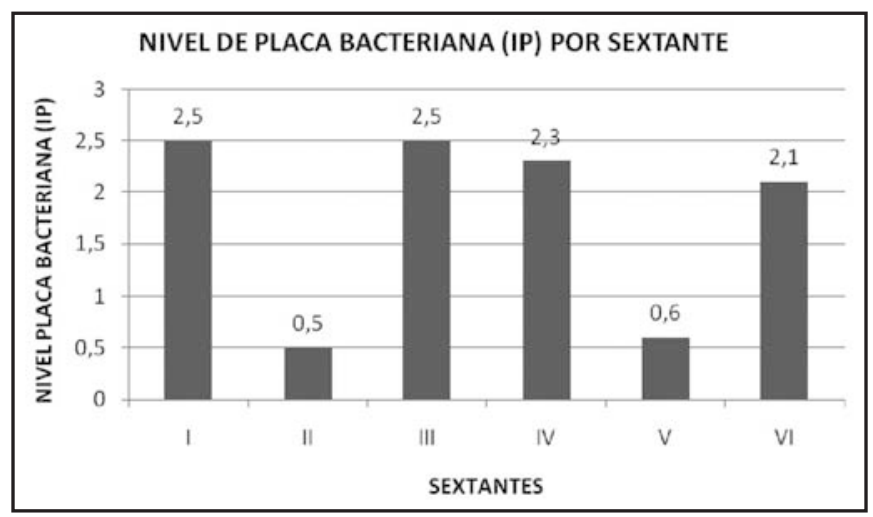

Gráfico 1.

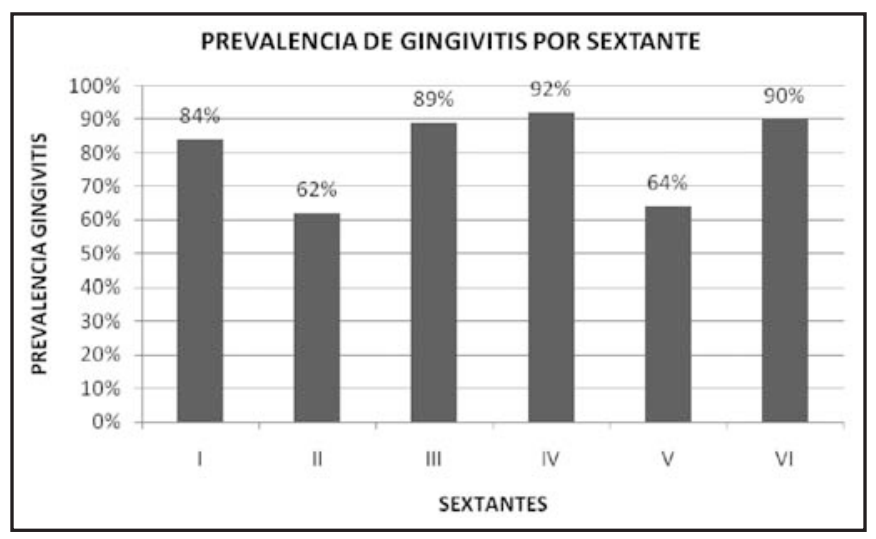

Gráfico 2.

\section{TABLA 1.- MODELO DE REGRESIÓN PARA MEDIDAS REPETIDAS DE LOS FACTORES DE RIESGO ASOCIADOS CON GINGIVITIS POR SEXTANTE}

\begin{tabular}{|c|c|}
\hline Variable & Valor $\mathrm{p}$ \\
\hline Género..... & 0,8307 \\
\hline Maloclusión & 0,8895 \\
\hline Tipo de maloclusión .............................. & 0,3156 \\
\hline 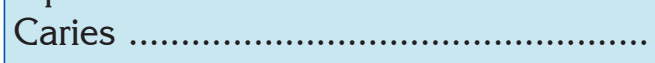 & $0,0014 *$ \\
\hline Frecuencia cepillado.. & 0,1046 \\
\hline Responsable cepillado ...... & 0,7458 \\
\hline Nivel de placa bacteriana ..................... & $<0,0001^{*}$ \\
\hline
\end{tabular}

\section{DISCUSIÓN}

Los estudios epidemiológicos han demostrado una gran variación en la prevalencia de gingivitis en niños y adolescentes con rangos que varían de un 35\% a $100 \%(2,7,9,17-19)$, esta información concuerda con los valores obtenidos en este estudio de preescolares en que la prevalencia es de 93,1\%. Esta diferencias se deben a los diferentes métodos diagnósticos utilizados en los estudios, lo que afecta la comparación entre estos.

Nuestros resultados difieren con los encontrados en el estudio de Feldens (9) en niños y niñas brasileños, a pesar que se utilizó el mismo criterio diagnóstico. La prevalencia encontrada en su estudio fue de un $72 \%$ a los 4 años. Esta diferencia podría deberse a que en nuestra población no habían sido intervenido previamente.

Los sitios gingivales más afectados fueron los posteroinferiores, seguidos por los posterosuperiores, similar a lo descrito tradicionalmente en la literatura científica (2, 9, 17, 21). Esto puede ser justificado debido a la dificultad de acceso a esas regiones de la boca durante el cepillado, especialmente en niños con edad preescolar (22).

Al evaluar los factores de riesgo asociados a gingivitis en niños preescolares se encontró evidencia estadísticamente significativa para relacionar nivel de pla- 
ca bacteriana, medido a través de IHO-S y presencia de gingivitis por sextante; lo que se contradice con otros estudios $(6,10,12,14,23)$ en donde la cantidad de placa bacteriana no se relaciona con el desarrollo de gingivitis en niños. Esta diferencia puede deberse a los diferentes criterios diagnósticos utilizados, ya en que similares a nuestro estudio, si existe una asociación significativa $(9,24)$.

Nuestro estudio también encontró asociación entre gingivitis y caries dental, la que guarda relación con el nivel de placa bacteriana presente. La presencia de caries inerproximales produce una mayor acumulación de placa bacteriana en estos sitios, que predispone a la pérdida de adherencia epitelial por el traumatismo que causa la impactación de alimentos $(25,26)$.

No encontramos relación directa entre gingivitis y cepillado nocturno, o responsable del cepillado. Esto se debe a los sesgos de información propios de cualquier encuesta o entrevista y que tiene que ver con la deseabilidad social, es decir, responden lo que creen que es correcto o lo que el profesional desea escuchar, ocultando la información verdadera. Además podría explicarse porque el tutor del niño, al momento del ingreso, no ha recibido enseñanza de técnica de cepillado, lo que ocasionaría que el adulto no pudiera realizar una técnica de cepillado capaz de remover adecuadamente la placa bacteriana.

Algunos estudios muestran una asociación significativa entre la ausencia de espacios interdentales y la presencia de gingivitis (27), especialmente en el sector anterosuperior (9). En nuestro estudio no detectamos diferencias, esto puede deberse a que sólo encontramos alteraciones en el sector anteroinferior, además la prevalencia de maloclusión encontrada fue baja.

\section{BIBLIOGRAFÍA}

1. Oh TJ, Eber R, Wang HL. Periodontal diseases in the child and adolescent. J Clin Periodontol 2002;29:400-10.

2. Jenkins W, Papanou P. Epidemiology of periodontal disease in children and adolescents. Periodontology 2000. 2001;26:16-32.
3. AAPD. Treatment of plaque-induced gingivitis, chronic periodontitis and other clinical conditions. Manuel reference 2004;29(7):22533.

4. AAPD. Periodontal disease of children and adolescent. Manuel reference 2004:189-96.

5. Moore W, Holdeman L, Smibert $\mathrm{R}$ et al. Bacteriology of experimental gingivitis in children. Infect Immun 1984;46:1-6.

6. Bimstein E, Matsson L. Growth and development consideration in the diagnosis of gingivitis and periodontitis in children. Pediatr Dent 1999;21: 186-91.

7. Stamm JW. Epidemiology of gingivitis. J Clin periodontal 1986;13:360-6

8. Modeer T, Wondimu B. Periodontal disease in children and adolescents. Dent Clin North Am. 2000;44:633-58.

9. Feldens E, Kramer P, Feldens C, Ferrerira S. Distribution of plaque and gingivitis and associated factors in 3 to 5 years old Brazilian Children. J Dent Child 2006;23:4-10.

10. Matson L, Goldberg P. Gingival inflammatory reaction in children at different ages. J Clin Periodontol 1985;12:98-103.

11. Bimstein E, Lustmann J, Soskolne WA. A clinical and histometric study of gingivitis associated with the human deciduous and permanent dentition. J Periodontol 1985;56:293-6.

12. Ramberg P, Lindhe J, Graffar A. Plaque and gingivitis in the deciduous and permanent dentition. J Clin Periodontol 1994;21:490-6.

13. Matson L. Development of gingivitis in pre-school children and young adults. J Clin Periodontol 1978; 5:24-34.

14. Bimstein E, Ebersole JL. The age-dependent reaction of the periodontal tissues to dental plaque. J Dent Child 1989;56:358-62. 
15. Mestrinho $\mathrm{H}$, Toledo O. Prevalence and distribution of gingivitis in Brazilian preschool children. Int Assoc Pediatr Dent 2001;18:121.

16. Bimstein E, Matson L, Soskolne A, Lustmann J. Histologic characteristics of the gingival associated with the primary and permanent teeth of children. Pediatr Dent 1994;16(3):206-10.

17. Hugoson A, Koch G, Rylander H. Prevalence and distribution of gingivitis - periodontitis in children and adolescents. Swed Dent J 1981;5:91-103.

18. Sayegh A, Dini EL, Holt RD. Relationship of socioeconomic background to oral hygiene, gingival status and dental caries in children. J Int Acad Period 2002;4(1):128-30.

19. Prevalencia y factores de riesgo asociados a enfermedad periodontal en preescolares de la ciudad de México. Gac Méd Méx 2005;141(3):185-9.

20. Massler M, Schour I, Choppa B. The P-M-A Index of gingivitis. J Dent Res 1949:28:634.

21. Mackler SB, Crawford JJ. Plaque development and gingivitis in primary dentition. $\mathrm{J}$ Periodontol 1973;44:18-24.

22. Ramberg P, Axelsson p, Lindhe J. Plaque formation at healthy and inflamed gingival sites in young individuals. J Clin Periodontol 1995; 22:85-8.
23. Matsson L. Factors influencing the susceptibility to gingiviti during chilfhood: A review. Int Clin Pediatr Dent 1993;3:119-27.

24. Spencer AJ, Beighton D, Higgins TJ. Periodontal disease in five and six-year old children. J Periodontol 1983;54:19-22.

25. Bimstein E. Frecuency of alveolar bone lose adjacent to proximal caries in primary molar and healing due to restoration óf the teeth. Pediatr Dent 1992;14:30-3.

26. Allison PJ, Schwartz S. Interproximal contact points and proximal caries in posterior primary teeth. Pediatr Dent 2003 Jul-Aug;25(4):33440.

27. Silness J, Roynstrand T. Effects on dental health of spacing of teeth in anterior segments. $\mathrm{J}$ Clin Periodontol 1984;11:387-98.

\section{CORRESPONDENCIA}

Carlos Zaror Sánchez

Manuel Montt, 112, 4 piso.

Dpto. Odontología Integral of. 420

Facultad de Medicina, Universidad de la Frontera Temuco, Chile

email: czaror@ufro.cl 